\title{
Population Data on Finland 1900-1991 ${ }^{1}$
}

UPDATED BY ANNELI MIETTINEN

\section{Resident population on 31 December}

\begin{tabular}{lccccr}
\hline & & \multicolumn{2}{c}{ Urban municipalities } & \multicolumn{2}{c}{ Rural municipalities } \\
& Whole country & number & $\%$ & 2322600 & 87.5 \\
\hline 1900 & 2655900 & 333400 & 12.5 & 2511200 & 85.3 \\
1910 & 2943400 & 432200 & 14.7 & 2640200 & 83.9 \\
1920 & 3147600 & 507400 & 16.1 & 2747700 & 79.4 \\
1930 & 3462700 & 715000 & 20.6 & 2703900 & 73.2 \\
1940 & 3695600 & 991700 & 26.8 & 2727400 & 67.7 \\
1950 & 4029800 & 1302400 & 32.3 & 2739200 & 61.6 \\
1960 & 4446200 & 1707000 & 38.4 & 2258000 & 49.1 \\
1970 & 4598300 & 2340300 & 50.9 & 1922700 & 40.2 \\
1980 & 4787800 & 2865100 & 59.8 & 1972800 & 40.2 \\
1985 & 4910700 & 2937900 & 59.8 & 1882700 & 38.2 \\
1986 & 4925600 & 3042900 & 61.8 & 1886200 & 38.2 \\
1987 & 4938600 & 3052400 & 61.8 & 1894800 & 38.2 \\
1988 & 4954400 & 3059500 & 61.8 & 1907400 & 38.3 \\
1989 & 4974400 & 3067000 & 61.7 & 1918700 & 38.4 \\
1990 & 4998500 & 3079800 & 61.6 & 1929300 & 38.4 \\
$1991 *$ & 5029300 & 3100000 & 61.6 & & \\
\hline
\end{tabular}

\section{Age structure}

\begin{tabular}{lccccccccr}
\hline & \multicolumn{3}{c}{ Whole population } & \multicolumn{3}{c}{ Men } & \multicolumn{3}{c}{ Women } \\
& $0-14$ & $15-64$ & $65-$ & $0-14$ & $15-64$ & $65-$ & $0-14$ & $15-64$ & $65-$ \\
& $\%$ & $\%$ & $\%$ & $\%$ & $\%$ & $\%$ & $\%$ & $\%$ & $\%$ \\
\hline 1900 & 35.0 & 59.6 & 5.4 & 35.9 & 59.4 & 4.7 & 34.3 & 59.7 & 6.0 \\
1910 & 35.6 & 58.6 & 5.8 & 36.7 & 58.3 & 5.0 & 34.6 & 59.0 & 6.4 \\
1920 & 33.4 & 60.7 & 5.9 & 34.8 & 60.0 & 5.2 & 32.0 & 61.3 & 6.7 \\
1930 & 29.4 & 64.3 & 6.3 & 30.6 & 64.1 & 5.3 & 28.3 & 64.5 & 7.2 \\
1940 & 26.9 & 66.7 & 6.4 & 28.2 & 66.5 & 5.3 & 25.7 & 66.9 & 7.4 \\
1950 & 30.0 & 63.3 & 6.7 & 32.0 & 62.8 & 5.2 & 28.1 & 63.9 & 8.0 \\
1960 & 30.1 & 62.4 & 7.4 & 31.9 & 62.3 & 5.7 & 28.5 & 62.5 & 8.9 \\
1970 & 24.4 & 66.3 & 9.3 & 25.7 & 67.1 & 7.2 & 23.0 & 65.7 & 11.3 \\
1980 & 20.2 & 67.8 & 12.0 & 21.4 & 69.7 & 8.9 & 19.1 & 66.0 & 14.9 \\
1985 & 19.4 & 68.0 & 12.6 & 20.5 & 70.4 & 9.1 & 18.4 & 65.8 & 15.8 \\
1986 & 19.3 & 67.9 & 12.8 & 20.4 & 70.3 & 9.3 & 18.3 & 65.6 & 16.1 \\
1987 & 19.3 & 67.8 & 12.9 & 20.4 & 70.3 & 9.4 & 18.3 & 65.4 & 16.3 \\
1988 & 19.4 & 67.5 & 13.1 & 20.4 & 70.0 & 9.5 & 18.4 & 65.1 & 16.5 \\
1989 & 19.3 & 67.4 & 13.3 & 20.4 & 69.9 & 9.7 & 18.4 & 64.9 & 16.7 \\
1990 & 19.3 & 67.2 & 13.5 & 20.3 & 69.8 & 9.9 & 18.3 & 64.8 & 16.9 \\
$1991^{*}$ & 19.2 & 67.2 & 13.6 & 20.2 & 69.8 & 10.0 & 18.2 & 64.7 & 17.0 \\
\hline
\end{tabular}

1 The data are based on the statistics of Statistics Finland, Helsinki.

* Preliminary data 
Figure 1. Age structure in $1900,1950,1990$ and 2010

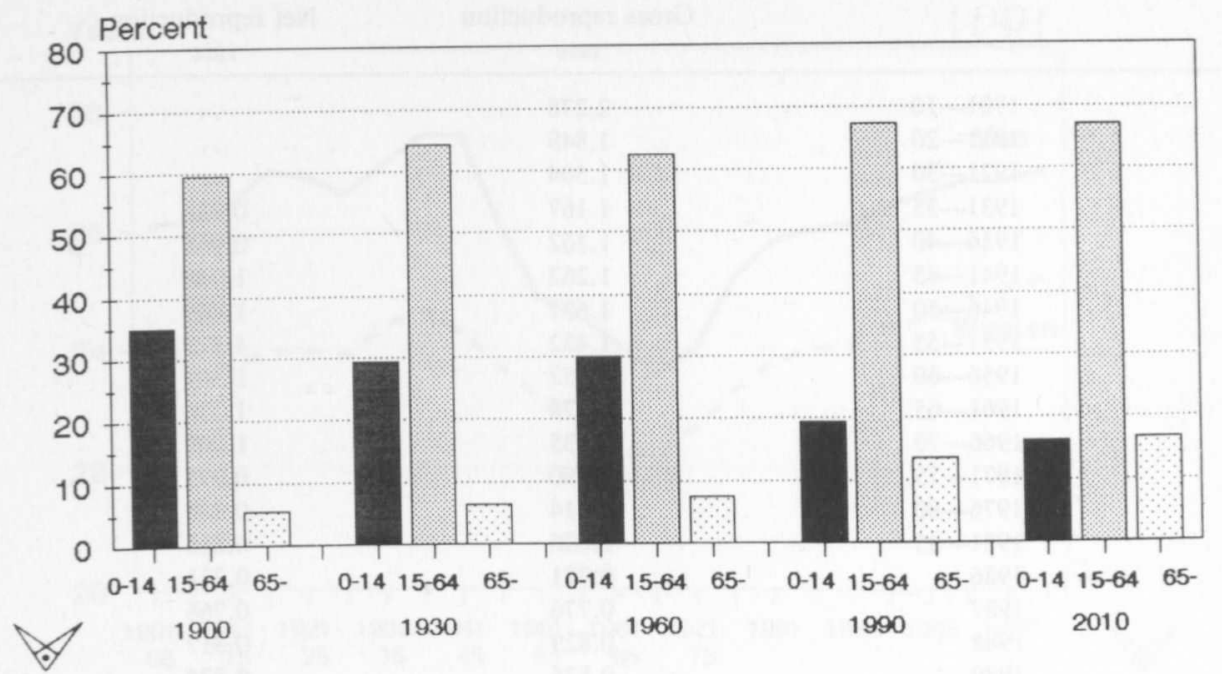

Age-specific fertility rate

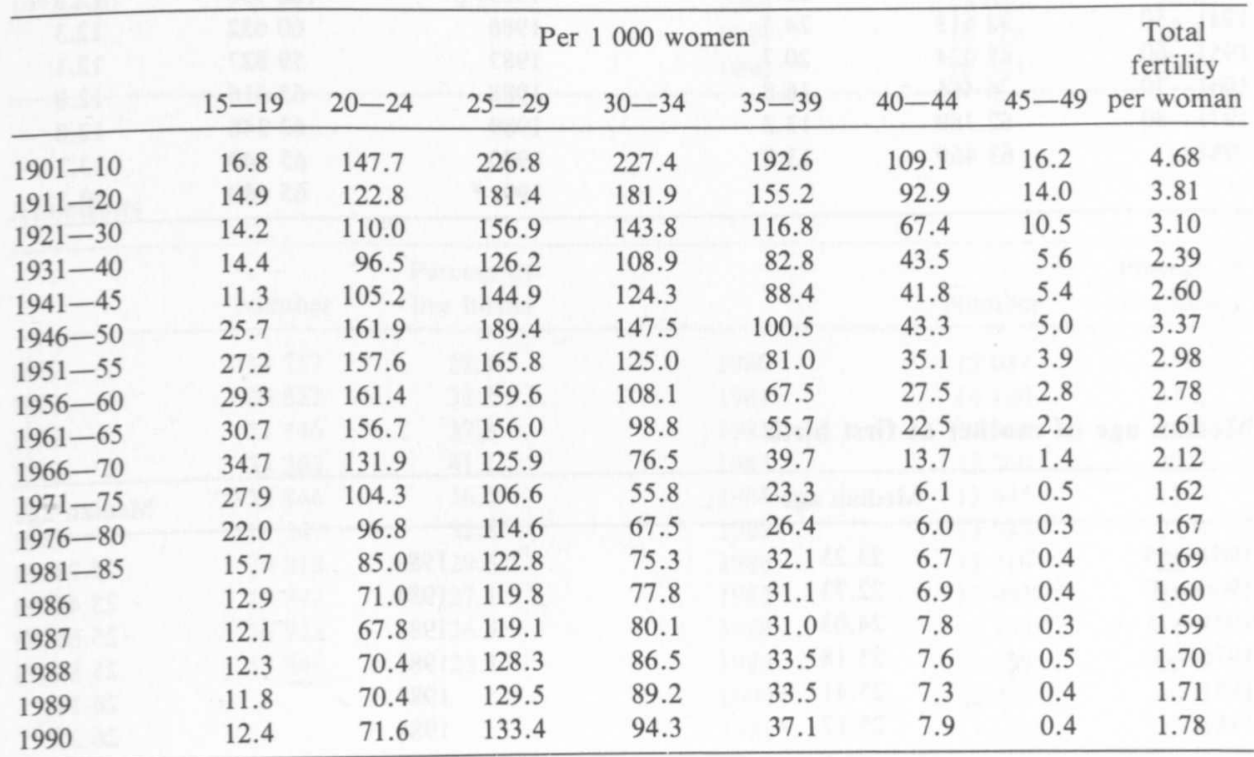




\begin{tabular}{lcc}
\hline & $\begin{array}{c}\text { Gross reproduction } \\
\text { rate }\end{array}$ & $\begin{array}{c}\text { Net reproduction } \\
\text { rate }\end{array}$ \\
\hline $1901-10$ & 2.278 & $\ldots$ \\
$1911-20$ & 1.849 & $\ldots$ \\
$1921-30$ & 1.504 & $\ldots$ \\
$1931-35$ & 1.167 & 0.958 \\
$1936-40$ & 1.162 & 0.962 \\
$1941-45$ & 1.262 & 1.048 \\
$1946-50$ & 1.637 & 1.469 \\
$1951-55$ & 1.452 & 1.373 \\
$1956-60$ & 1.357 & 1.301 \\
$1961-65$ & 1.276 & 1.236 \\
$1966-70$ & 1.035 & 1.009 \\
$1971-75$ & 0.790 & 0.773 \\
$1976-80$ & 0.814 & 0.801 \\
$1981-85$ & 0.826 & 0.815 \\
1986 & 0.781 & 0.771 \\
1987 & 0.776 & 0.766 \\
1988 & 0.829 & 0.817 \\
1989 & 0.836 & 0.824 \\
1990 & 0.872 & 0.860 \\
\hline
\end{tabular}

\section{Live births}

\begin{tabular}{lccccc}
\hline & $\begin{array}{c}\text { Annual } \\
\text { mean }\end{array}$ & $\begin{array}{c}\text { Per } 1000 \\
\text { of mean } \\
\text { population }\end{array}$ & & & $\begin{array}{c}\text { Per 1 000 } \\
\text { of mean } \\
\text { population }\end{array}$ \\
\hline $1901-10$ & 90292 & 32.4 & 1982 & 66106 & 13.7 \\
$1911-20$ & 83045 & 27.0 & 1983 & 66892 & 13.8 \\
$1921-30$ & 78184 & 23.6 & 1984 & 65076 & 13.3 \\
$1931-40$ & 70584 & 19.7 & 1985 & 62796 & 12.8 \\
$1941-50$ & 92613 & 24.3 & 1986 & 60632 & 12.3 \\
$1951-60$ & 88024 & 20.7 & 1987 & 59827 & 12.1 \\
$1961-70$ & 76466 & 16.8 & 1988 & 63316 & 12.8 \\
$1971-80$ & 62789 & 13.3 & 1989 & 63348 & 12.8 \\
1981 & 63469 & 13.2 & 1990 & 65549 & 13.1 \\
& & & $1991 *$ & 65680 & 13.1 \\
\hline
\end{tabular}

Median age of mother at first birth

\begin{tabular}{|c|c|c|c|}
\hline$x^{2}$ & Median age & & Median age \\
\hline $1961-65$ & 23.25 & 1983 & 25.34 \\
\hline $1966-70$ & 22.73 & 1984 & 25.46 \\
\hline $1971-75$ & 24.03 & 1985 & 25.66 \\
\hline $1976-80$ & 25.18 & 1986 & 25.86 \\
\hline 1981 & 25.41 & 1987 & 26.14 \\
\hline 1982 & 25.17 & 1988 & 26.27 \\
\hline
\end{tabular}


Figure 2. Median age at first marriage

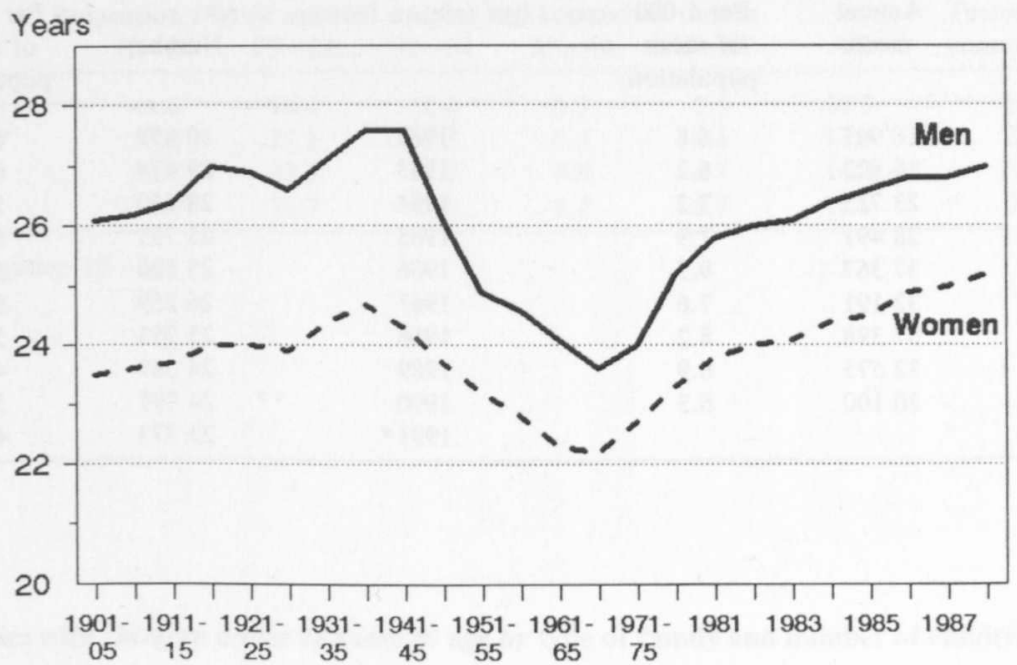

Illegitimate live births

\begin{tabular}{|c|c|c|c|c|c|}
\hline & $\begin{array}{c}\text { Annual } \\
\text { mean }\end{array}$ & $\begin{array}{l}\text { Percent } \\
\text { of all live } \\
\text { births }\end{array}$ & & Number & $\begin{array}{l}\text { Percent } \\
\text { of all live } \\
\text { births }\end{array}$ \\
\hline $1901-10$ & 6120 & 6.8 & 1981 & 8431 & 13.3 \\
\hline $1911-20$ & 6573 & 6.8 & 1982 & 9007 & 13.6 \\
\hline $1921-30$ & 6606 & 8.4 & 1983 & 9386 & 14.0 \\
\hline $1931-40$ & 5349 & 7.6 & 1984 & 9825 & 15.1 \\
\hline $1941-50$ & 5629 & 6.1 & 1985 & 10292 & 16.4 \\
\hline $1951-60$ & 3819 & 4.3 & 1986 & 10931 & 18.0 \\
\hline $1961-70$ & 3621 & 4.7 & 1987 & 11467 & 19.2 \\
\hline \multirow[t]{3}{*}{$1971-80$} & 6182 & 9.8 & 1988 & 13046 & 20.6 \\
\hline & & & 1989 & 14516 & 22.9 \\
\hline & & & 1990 & 16543 & 25.2 \\
\hline
\end{tabular}

\section{Abortions}

\begin{tabular}{lccccc}
\hline & Number & $\begin{array}{c}\text { Percent of } \\
\text { live births }\end{array}$ & Number & $\begin{array}{c}\text { Percent of } \\
\text { live births }\end{array}$ \\
\hline 1970 & 14757 & 22.9 & 1980 & 15037 & 23.8 \\
1971 & 20622 & 33.8 & 1981 & 14120 & 22.2 \\
1972 & 22146 & 37.5 & 1982 & 13861 & 21.0 \\
1973 & 23362 & 41.1 & 1983 & 13360 & 20.0 \\
1974 & 22846 & 36.5 & 1984 & 13645 & 21.0 \\
1975 & 21547 & 32.8 & 1985 & 13833 & 22.0 \\
1976 & 19818 & 29.6 & 1986 & 13319 & 22.0 \\
1977 & 17772 & 27.1 & 1987 & 12995 & 21.7 \\
1978 & 16928 & 26.4 & 1988 & 12658 & 19.2 \\
1979 & 15849 & 25.1 & $1990^{*}$ & 12439 & 20.0 \\
& & & $1991^{*}$ & 11660 & 19.0 \\
\hline
\end{tabular}


Marriages

\begin{tabular}{lccccc}
\hline & $\begin{array}{c}\text { Annual } \\
\text { mean }\end{array}$ & $\begin{array}{c}\text { Per 1 000 } \\
\text { of mean } \\
\text { population }\end{array}$ & & Number & $\begin{array}{c}\text { Per 1 000 } \\
\text { of mean } \\
\text { population }\end{array}$ \\
\hline $1901-10$ & 18947 & 6.8 & 1982 & 30459 & 6.3 \\
$1911-20$ & 18922 & 6.2 & 1983 & 29474 & 6.1 \\
$1921-30$ & 23725 & 7.2 & 1984 & 28550 & 5.8 \\
$1931-40$ & 28491 & 7.9 & 1985 & 25751 & 5.3 \\
$1941-50$ & 37367 & 9.5 & 1986 & 25820 & 5.2 \\
$1951-60$ & 32191 & 7.6 & 1987 & 26259 & 5.3 \\
$1961-70$ & 37398 & 8.2 & 1988 & 25933 & 5.2 \\
$1971-80$ & 32575 & 6.9 & 1989 & 24569 & 4.9 \\
1981 & 30100 & 6.3 & 1990 & 24997 & 5.0 \\
& & & $1991^{*}$ & 23573 & 4.7 \\
\hline
\end{tabular}

Median age at first marriage

\begin{tabular}{lcclcc}
\hline & Men & Women & & Men & Women \\
\hline $1901-05$ & 26.1 & 23.5 & $1961-65$ & 24.1 & 22.3 \\
$1906-10$ & 26.2 & 23.6 & $1966-70$ & 23.6 & 22.2 \\
$1911-15$ & 26.4 & 23.7 & $1971-75$ & 24.0 & 22.7 \\
$1916-20$ & 27.0 & 24.0 & $1976-80$ & 25.2 & 23.3 \\
$1921-25$ & 26.9 & 24.0 & $1981-85$ & 26.2 & 24.2 \\
$1926-30$ & 26.6 & 23.9 & 1986 & 26.8 & 24.9 \\
$1931-35$ & 27.1 & 24.4 & 1987 & 26.8 & 25.0 \\
$1936-40$ & 27.6 & 24.7 & 1988 & 27.0 & 25.2 \\
$1941-45$ & 27.6 & 24.3 & & & \\
$1946-50$ & 26.1 & 23.7 & & & \\
$1951-55$ & 24.9 & 23.2 & & & \\
$1956-60$ & 24.6 & 22.8 & & &
\end{tabular}

\section{Divorces}

\begin{tabular}{lccccc}
\hline & $\begin{array}{c}\text { Annual } \\
\text { mean }\end{array}$ & $\begin{array}{c}\text { Per 1 000 } \\
\text { of mean } \\
\text { population }\end{array}$ & & Number & $\begin{array}{c}\text { Per } 1000 \\
\text { of mean } \\
\text { population }\end{array}$ \\
\hline $1951-60$ & 3584 & 0.84 & 1984 & 9652 & 1.97 \\
$1961-70$ & 4860 & 1.06 & 1985 & 9064 & 1.85 \\
$1971-75$ & 8727 & 1.87 & 1986 & 9742 & 1.98 \\
$1976-80$ & 10051 & 2.12 & 1987 & 10110 & 2.05 \\
1981 & 9497 & 1.98 & 1988 & 12146 & 2.46 \\
1982 & 9705 & 2.01 & 1989 & 14365 & 2.89 \\
1983 & 9770 & 2.01 & 1990 & 13127 & 2.63 \\
& & & $1991^{*}$ & 12791 & 2.55 \\
\hline
\end{tabular}


Consensual unions

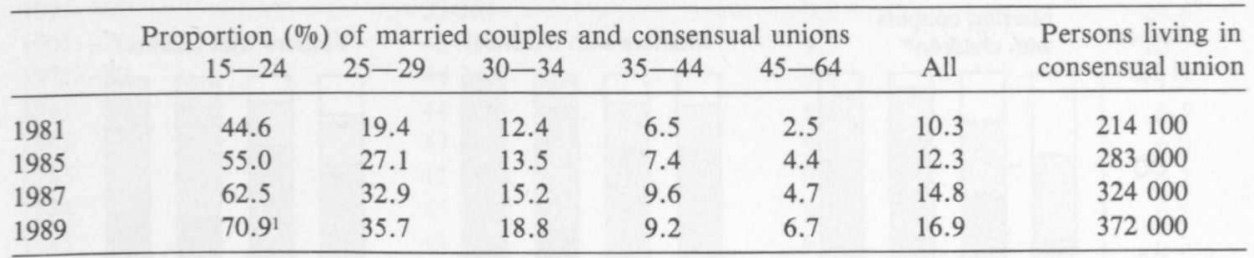

${ }^{1}$ Age group 18-24

Families with children under 18 years of age by type of family and number of children (percent)

Married couples with children ${ }^{1}$

$\begin{array}{lcccrc} & 1 & 2 & 3 & 4+ & \text { Number } \\ 1960 & 34.4 & 30.7 & 17.5 & 17.4 & 601542 \\ 1970 & 40.2 & 33.9 & 15.5 & 10.4 & 602076 \\ 1975 & 44.9 & 37.0 & 12.6 & 5.5 & 593346 \\ 1980 & 44.6 & 41.1 & 11.1 & 3.2 & 588373 \\ 1984 & 43.2 & 41.7 & 12.0 & 3.2 & 569568 \\ 1987 & 41.1 & 42.3 & 13.0 & 3.6 & 545698 \\ 1990 & 40.3 & 41.6 & 14.1 & 4.0 & 534665 \\ 1990^{2} & 38.9 & 42.4 & 14.5 & 4.1 & 490965\end{array}$

Mothers with children

$\begin{array}{lccccr} & 1 & 2 & 3 & 4+ & \text { Number } \\ 1960 & 61.1 & 23.0 & 9.2 & 6.7 & 67381 \\ 1970 & 60.7 & 23.8 & 9.5 & 6.0 & 66303 \\ 1975 & 62.4 & 25.7 & 8.3 & 3.6 & 72359 \\ 1980 & 67.7 & 25.3 & 5.6 & 1.4 & 88896 \\ 1984 & 70.1 & 24.4 & 4.6 & 0.9 & 88419 \\ 1987 & 69.0 & 25.2 & 4.8 & 1.0 & 86377 \\ 1990 & 66.8 & 26.5 & 5.5 & 1.2 & 93669 \\ 1990^{2} & 65.6 & 27.2 & 5.9 & 1.3 & 78869\end{array}$

Fathers with children

$\begin{array}{lccccr} & 1 & 2 & 3 & 4+ & \text { Number } \\ 1960 & 56.7 & 24.8 & 10.3 & 8.2 & 9123 \\ 1970 & 61.1 & 24.3 & 9.0 & 5.6 & 8656 \\ 1975 & 64.0 & 24.6 & 8.4 & 3.0 & 10386 \\ 1980 & 71.1 & 23.0 & 4.8 & 1.1 & 11463 \\ 1984 & 74.9 & 21.3 & 3.3 & 0.6 & 11573 \\ 1987 & 73.8 & 22.0 & 3.7 & 0.6 & 11918 \\ 1990 & 72.5 & 22.9 & 3.8 & 0.7 & 13240 \\ 1990^{2} & 71.7 & 23.4 & 4.1 & 0.8 & 11074\end{array}$

Couples living in consensual union with children

\begin{tabular}{llllll}
$1990^{2}$ & 60.0 & 30.2 & 7.9 & 1.9 & 59827 \\
\hline
\end{tabular}

1 Incl. not-married couples with children under 18 years

2 The number of consensual unions has been calculated in a new way 
Figure 3. Families with children under 18 years of age

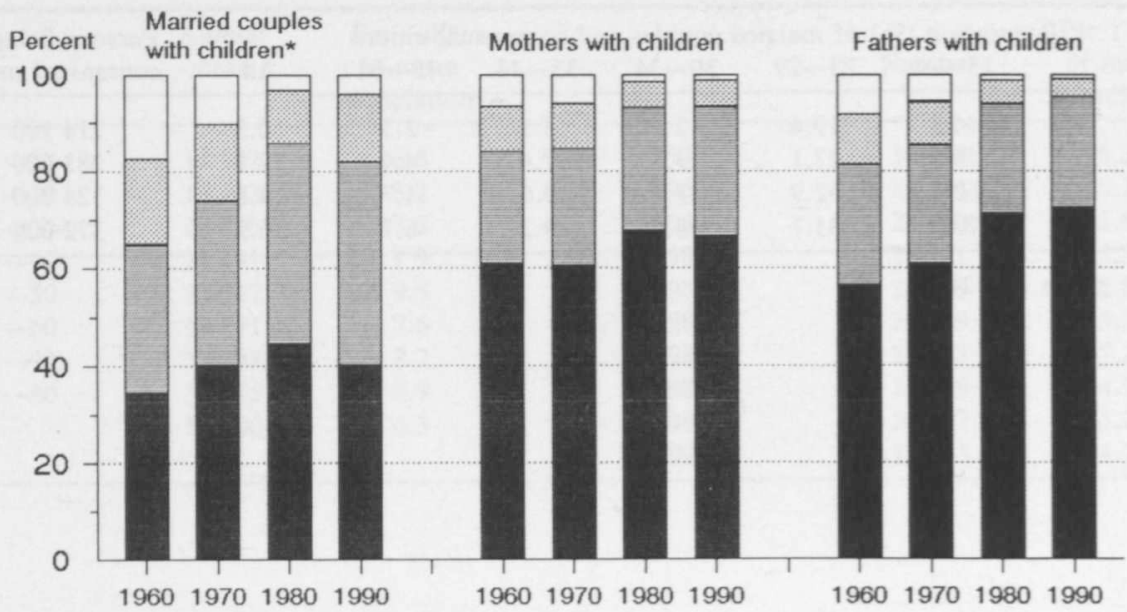

a $1 \square 2 \square 3 \square 4+$ Children

* including couples living in consensual unions

Families with children under 18 years of age by type of family

\begin{tabular}{lcccc}
\hline & $\begin{array}{c}\text { Married couples } \\
\text { with children } \\
\%\end{array}$ & $\begin{array}{c}\text { Mothers } \\
\text { with children } \\
\%\end{array}$ & $\begin{array}{c}\text { Fathers } \\
\text { with children } \\
\%\end{array}$ & Number \\
\hline 1960 & 88.7 & 10.0 & 1.3 & 678046 \\
1970 & 88.9 & 9.8 & 1.3 & 677035 \\
1975 & 87.8 & 10.7 & 1.5 & 676091 \\
1978 & 85.4 & 13.0 & 1.6 & 697145 \\
1980 & 85.4 & 12.9 & 1.7 & 688732 \\
1982 & 85.1 & 13.2 & 1.7 & 682865 \\
1984 & 85.1 & 13.2 & 1.7 & 669560 \\
1985 & 85.0 & 13.2 & 1.8 & 659870 \\
1987 & 84.7 & 13.4 & 1.9 & 643993 \\
1990 & 83.3 & 14.6 & 2.1 & 641574 \\
$1990^{2}$ & 85.9 & 12.3 & 1.7 & 640735 \\
\hline
\end{tabular}

1 Incl. not-married couples with children under 18 years

2 The number of consensual unions has been calculated in a new way

\section{Deaths}

\begin{tabular}{lccc}
\hline & $\begin{array}{c}\text { Annual mean/ } \\
\text { Number }\end{array}$ & $\begin{array}{c}\text { Per } 1000 \\
\text { of mean } \\
\text { population }\end{array}$ & $\begin{array}{c}\text { Infant mortality } \\
\text { per } 1000 \text { live } \\
\text { births }\end{array}$ \\
\hline $1901-10$ & 52099 & 18.7 & 123.8 \\
$1911-20$ & 58284 & 19.0 & 111.8 \\
$1921-30$ & 49573 & 14.9 & 91.9 \\
$1931-40$ & 50222 & 14.0 & 72.0
\end{tabular}




\begin{tabular}{lrrr}
$1941-50$ & 51838 & 13.6 & 56.0 \\
$1951-60$ & 39381 & 9.3 & 28.6 \\
$1961-70$ & 43494 & 9.5 & 16.7 \\
$1971-80$ & 44243 & 9.4 & 11.3 \\
1981 & 44404 & 9.3 & 6.5 \\
1982 & 43408 & 9.0 & 6.0 \\
1983 & 45388 & 9.3 & 6.2 \\
1984 & 45098 & 9.2 & 6.5 \\
1985 & 48198 & 9.8 & 6.3 \\
1986 & 47135 & 9.6 & 5.9 \\
1987 & 47949 & 9.7 & 6.2 \\
1988 & 49063 & 9.9 & 6.1 \\
1989 & 49110 & 9.9 & 6.0 \\
1990 & 50058 & 10.0 & $\ldots$ \\
$1991^{*}$ & 49271 & 9.8 & $\ldots$ \\
\hline
\end{tabular}

Mean expectation of life at birth

\begin{tabular}{lccccc}
\hline & Men & Women & & Men & Women \\
\hline $1911-20^{1}$ & 43.4 & 49.1 & $1966-70$ & 65.9 & 73.6 \\
$1921-30$ & 50.7 & 55.1 & $1971-75$ & 66.7 & 75.2 \\
$1931-40^{2}$ & 54.5 & 59.6 & $1976-80$ & 68.5 & 77.2 \\
$1941-45^{2}$ & 54.6 & 61.1 & $1981-85$ & 70.1 & 78.4 \\
$1946-50$ & 58.6 & 65.8 & 1986 & 70.5 & 78.7 \\
$1951-55$ & 63.4 & 69.8 & 1987 & 70.7 & 78.7 \\
$1956-60$ & 65.9 & 71.6 & 1988 & 70.7 & 78.7 \\
$1961-65$ & 65.4 & 72.6 & 1989 & 70.9 & 78.9 \\
& & & 1990 & 70.9 & 78.9 \\
\hline
\end{tabular}

1 Including deaths due to war operations.

2 Excluding deaths due to war operations.

Figure 4. Mean expectation of life at birth

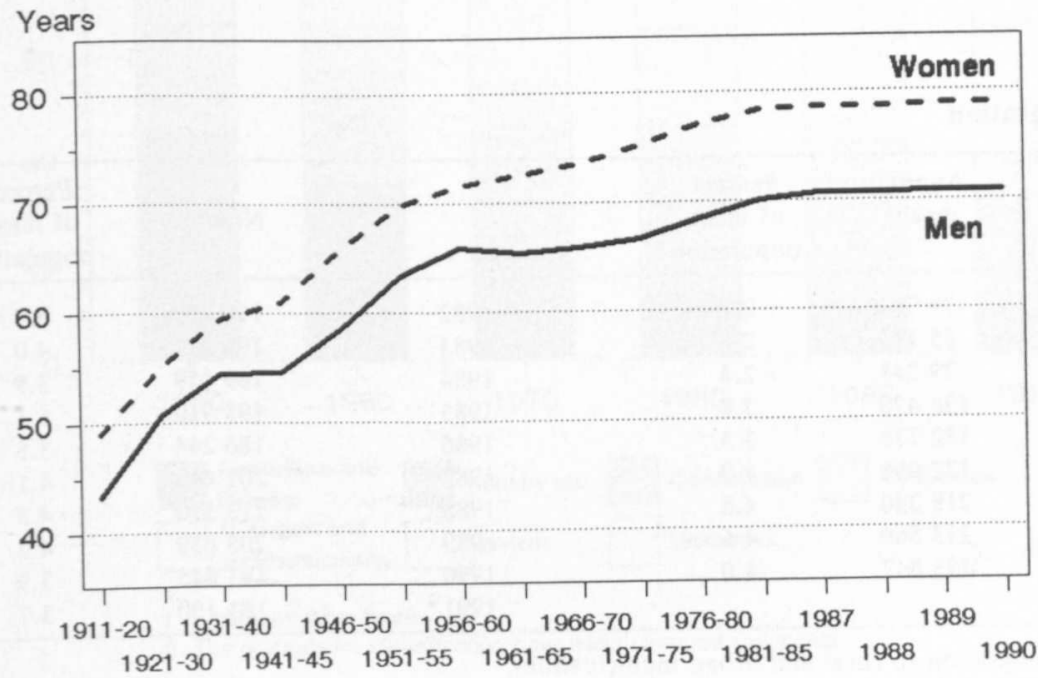


Excess of births over deaths

\begin{tabular}{lccccc}
\hline & Annual mean & $\begin{array}{c}\text { Per 1 000 } \\
\text { of mean } \\
\text { population }\end{array}$ & & $\begin{array}{c}\text { Per } 1000 \\
\text { Number } \\
\text { mean } \\
\text { population }\end{array}$ \\
\hline $1901-10$ & 38194 & 13.7 & 1986 & 13497 & 2.7 \\
$1911-20$ & 24760 & 8.1 & 1987 & 11878 & 2.4 \\
$1921-30$ & 28611 & 8.7 & 1988 & 14253 & 2.9 \\
$1931-40$ & 20354 & 5.7 & 1989 & 15491 & 3.9 \\
$1941-50$ & 44272 & 10.7 & 1990 & 16409 & 3.3 \\
$1951-60$ & 48643 & 11.4 & & & \\
$1961-70$ & 32223 & 7.2 & & & \\
$1971-75$ & 16632 & 3.6 & & & \\
$1976-80$ & 20460 & 4.3 & & & \\
$1981-85$ & 19569 & 4.0 & & & \\
\hline
\end{tabular}

International migration

\begin{tabular}{|c|c|c|c|c|c|}
\hline & $\begin{array}{c}\text { Emigrants who } \\
\text { have obtained } \\
\text { a passport }\end{array}$ & $\begin{array}{l}\text { Per } 1000 \\
\text { of mean } \\
\text { population }\end{array}$ & & $\begin{array}{l}\text { Yearly net } \\
\text { migration }\end{array}$ & $\begin{array}{c}\text { Per } 1000 \\
\text { of mean } \\
\text { population }\end{array}$ \\
\hline $1901-10$ & 150832 & 5.4 & $1976-80$ & -7493 & -1.6 \\
\hline $1911-20$ & 67346 & 2.2 & $1981-85$ & +5347 & 1.1 \\
\hline $1921-30$ & 58559 & 1.8 & 1986 & +1658 & 0.3 \\
\hline \multirow[t]{2}{*}{$1931-40$} & 8844 & 0.2 & 1987 & +667 & 0.1 \\
\hline & & & 1988 & +1273 & 0.3 \\
\hline \multirow{2}{*}{\multicolumn{3}{|c|}{ Yearly net migration }} & 1989 & +3845 & 0.8 \\
\hline & & & 1990 & +7081 & 1.4 \\
\hline $1941-50$ & -4100 & -1.1 & $1991^{*}$ & +13847 & 2.8 \\
\hline $1951-60$ & -7003 & -1.5 & & & \\
\hline $1961-70$ & -17763 & -3.9 & & & \\
\hline $1971-75$ & +1936 & 0.4 & & & \\
\hline
\end{tabular}

\section{Internal migration}

\begin{tabular}{lccccc}
\hline & $\begin{array}{c}\text { Annual } \\
\text { mean }\end{array}$ & $\begin{array}{c}\text { Percent } \\
\text { of mean } \\
\text { population }\end{array}$ & Number & $\begin{array}{c}\text { Percent } \\
\text { of mean } \\
\text { population }\end{array}$ \\
\hline $1901-10$ & 74784 & 2.7 & 1982 & 190419 & 3.9 \\
$1911-20$ & 85111 & 2.8 & 1983 & 196070 & 4.0 \\
$1921-30$ & 79248 & 2.4 & 1984 & 189159 & 3.9 \\
$1931-39$ & 136470 & 3.8 & 1985 & 198910 & 4.1 \\
$1941-50$ & 132778 & 3.5 & 1986 & 186244 & 3.8 \\
$1951-60$ & 172098 & 4.0 & 1987 & 201448 & 4.1 \\
$1961-70$ & 219330 & 4.8 & 1988 & 213510 & 4.3 \\
$1971-80$ & 213569 & 4.6 & 1989 & 208639 & 4.2 \\
1981 & 193847 & 4.0 & 1990 & 193845 & 3.9 \\
& & & $1991^{*}$ & 183130 & 3.7 \\
\hline
\end{tabular}

1 Average in-migration to rural and urban municipalities. 
Economically active population by industry (percent)

\begin{tabular}{lrrrrrr}
\hline & 1950 & 1960 & 1970 & 1980 & $1985^{1}$ & $1990^{12}$ \\
\hline Agriculture and forestry, & & & & & & \\
$\quad$ hunting and fishing & 45.8 & 35.5 & 20.3 & 12.6 & 10.6 & 8.4 \\
Industry etc. & 20.8 & 21.6 & 25.9 & 26.3 & 24.3 & 22.6 \\
Construction & 6.3 & 8.7 & 8.3 & 7.1 & 7.4 & 8.3 \\
Commerce & 9.5 & 13.6 & 18.9 & 19.1 & 21.3 & 25.6 \\
Transport and communications & 5.4 & 6.3 & 7.1 & 7.9 & 7.6 & 7.2 \\
Services & 10.8 & 14.0 & 18.1 & 24.8 & 28.5 & 27.8 \\
Unknown & 1.4 & 0.3 & 1.4 & 2.2 & 0.3 & 0.1 \\
\hline & 100.0 & 100.0 & 100.0 & 100.0 & 100.0 & 100.0 \\
\hline
\end{tabular}

1 Employed labor force

2 The grounds for classification have been changed somewhat

Figure 5. Economically active population by industry

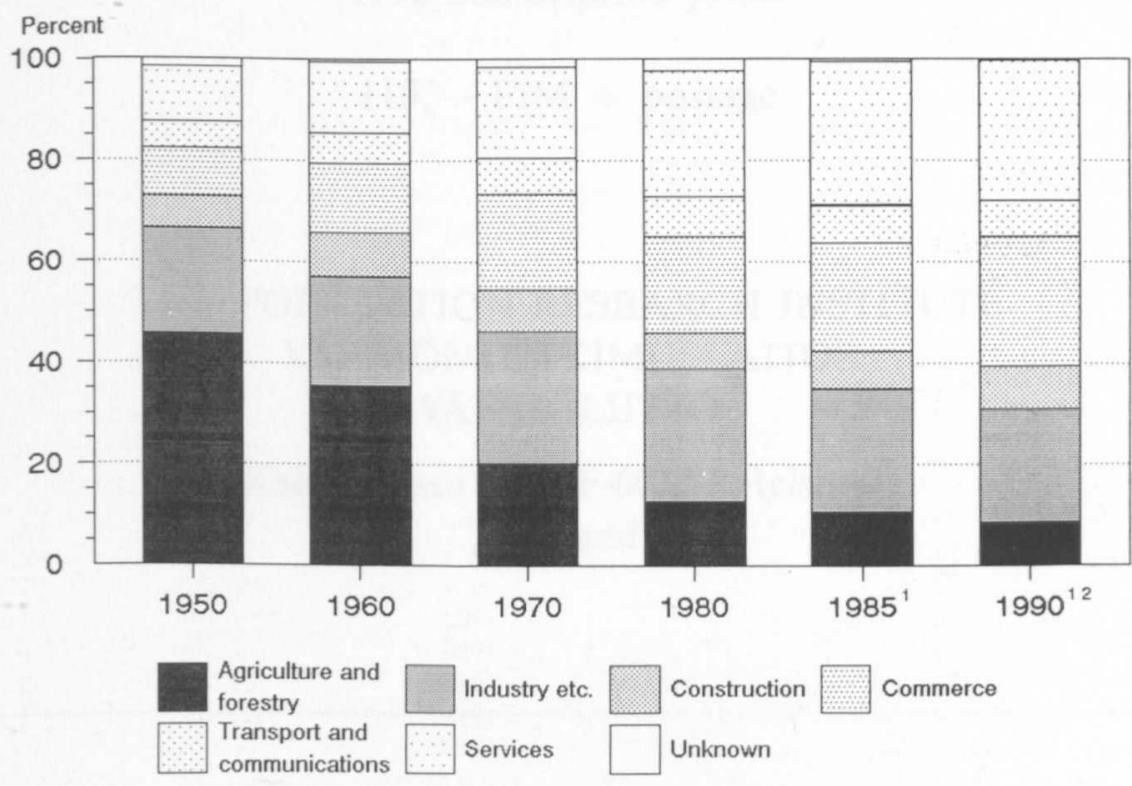

1 Employed labor force

2 The grounds for classification have been changed somewhat 


\section{Females in labor force $^{1}$}

The proportion

of females in labor

force (percent)
Female labor force as a percentage of female population of $15-74$ years

\begin{tabular}{llll}
\hline $1960-64$ & 44.0 & $1960-64$ & 56.2 \\
$1965-69$ & 43.3 & $1965-69$ & 55.1 \\
$1970-74$ & 44.7 & $1970-74$ & 57.9 \\
$1975-79$ & 46.3 & $1975-79$ & 59.9 \\
$1980-84$ & 47.3 & $1980-84$ & 63.0 \\
1985 & 47.9 & 1985 & 64.8 \\
1986 & 47.6 & 1986 & 64.5 \\
1987 & 47.6 & 1987 & 64.1 \\
1988 & 47.7 & 1988 & 64.1 \\
1989 & 47.6 & 1989 & 64.3 \\
1990 & 47.5 & 1990 & 63.9 \\
\hline
\end{tabular}

1 The data for 1981-87 have been corrected to correspond to the data of the revised Labour Force Survey from 1987 on. 